\title{
Vaccines against tropical parasitic diseases: a persisting answer to a persisting problem
}

\author{
David L Sacks
}

\section{Live whole-organism vaccines against Plasmodium falciparum malaria and cutaneous leishmaniasis remain the most uniformly effective vaccines against human parasitic diseases. These vaccines are discussed in terms of the requirement for persisting antigen to generate and maintain a protective response.}

\begin{abstract}
Tropical infectious diseases caused by para-
sites are major causes of illness in the poorest countries of Asia, Africa and Latin America. Among the tropical diseases with the highest burden, which are commonly referred to as 'neglected', 11 are caused by helminthic and protozoan parasites. These diseases, along with malaria, affect more than 1 billion people and cause more than 1 million deaths annually ${ }^{1}$. The even greater impact of these infections may be the chronic disabilities that they produce, such as malnutrition, anemia, cognitive defects and disfigurement, and the economic hardships that result from the cost of treatment and loss of worker productivity ${ }^{2}$.

The measures currently available to reduce the burden of tropical parasitic diseases are confined to drug-treatment programs and/ or to vector control. These interventions have resulted in selection for both resistant parasites and vectors, which, along with the high cost and low sustainability of the interventions, has reinforced the need for preventive vaccines. Unfortunately, there is as yet no safe, uniformly effective vaccine against any human parasitic infection. The development of the so-called 'antipoverty' vaccines ${ }^{2}$ must be considered one of the major unachieved goals of modern immunology. The absence of a commercial market remains a serious disincentive for industry to take on this effort, but even when product development partnerships were set up to oversee vaccine development through to proper human
\end{abstract}

David L. Sacks is at the Laboratory of Parasitic Diseases, National Institute of Allergy and Infectious Diseases, National Institutes of Health, Bethesda, Maryland, USA.

e-mail: dsacks@niaid.nih.gov trials, the goal of producing a highly effective vaccine has not been met. The greater impediments to vaccine development may be the gaps in our knowledge of the biology of these eukaryotic pathogens, their complexity as immunologic targets and their remarkable adaptability to immunologic pressure.

The hallmark of parasitic infections is their chronicity, which implies a certain capacity to avoid or delay sterilizing immunity. The adaptive strategies that protozoan and metazoan parasites use to evade immunity_-for example, antigenic variation, sequestration and immunosuppression-are driven in many parasites by their need to prolong their survival in the mammalian host in order to counteract their relatively low transmissibility to the arthropod vector on which their cyclical development depends. Thus, for a given antiparasite vaccine to succeed, it will have to outperform the immune response to natural, primary infection. This is fundamentally different from most licensed vaccines, which are designed to mimic the sterilizing response to natural infection without producing disease. It will be especially difficult for a vaccine to contend with protective antigens that display extensive allelic or somatic polymorphisms. Such targets would include the variant surface glycoprotein of African trypanosomes, the blood-stage malaria proteins expressed on the surface of merozoites or infected erythrocytes and the transialidase surface antigens of T. $c r u z i^{3}$.

There are, nonetheless, rare examples of antiparasite vaccines in humans that are remarkable for their success. Two vaccines in particular, the whole sporozoites that protect against $P$. falciparum malaria and the whole Leishmania major promastigotes that protect against cutaneous leishmaniasis, are the gold standards of acquired resistance against their respective diseases. In each case, live or live-attenuated organisms have been used. Although one clear advantage of whole-cell vaccines is their breadth of coverage against a multiplicity of antigens to better contend with parasite strain polymorphisms and host genetic restrictions, the more critical quality of the two vaccines, and the focus of this Commentary, is antigen persistence.

Whole-sporozoite vaccines against malaria Nearly 40 years ago it was observed that sterilizing immunity against $P$. falciparum could be achieved by exposing human volunteers to the bites of irradiated mosquitoes carrying sporozoites in their salivary glands ${ }^{4}$. The radiationattenuated parasites could not develop beyond the liver stages. These trials followed closely on ground-breaking studies using intravenous inoculation of irradiated $P$. bergei sporozoites in mice ${ }^{5}$. In both mice and humans, complete protection against infectious sporozoite challenge was dependent on the parasites being metabolically active and on a high dose exposure $(>1,000$ bites needed to achieve protection in people). A few of the human volunteers were still protected 23-42 weeks after their primary or secondary immunization ${ }^{6}$. Early on it seemed clear, however, that the inability to grow $P$. falciparum sporozoites in culture would preclude their use as a practical approach to vaccination. An era of subunit, pre-erythrocytic stage vaccine development ensued, in which both antibody-meditated and $\mathrm{T}$ cell-mediated responses were targeted, culminating with the recent phase III trials of the RTS,S malaria vaccine, a circumsporozoite protein-based subunit vaccine expressed in a hepatitis B-like particle ${ }^{7}$. In no case has the 


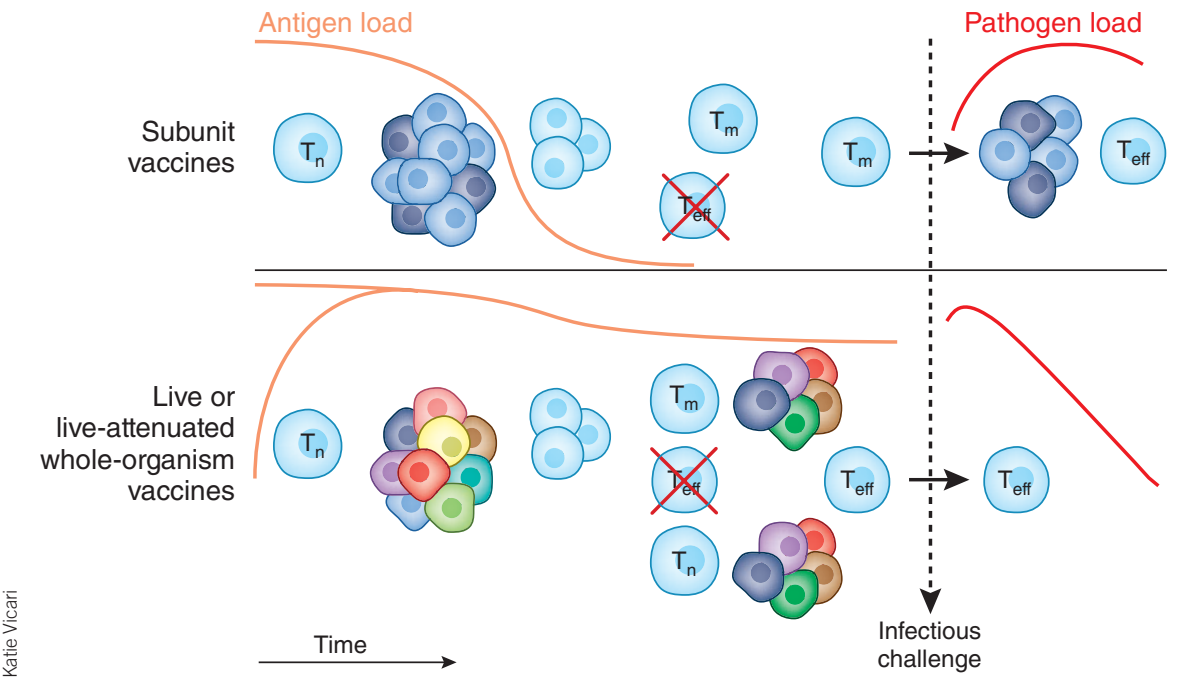

Figure 1 The role of antigen persistence in vaccine-induced protection. Expansion and contraction of antigen-specific $T$ cells from naive precursors $\left(T_{n}\right)$, and their differentiation into memory cell $\left(T_{m}\right)$ and effector cell $\left(T_{\text {eff }}\right)$ pools in response to subunit vaccines, or to live (for example, L. major) or liveattenuated (for example, irradiated sporozoites) whole-organism vaccines. Colors represent the relative breadth of the antigen-specific response. Effector T cells are short-lived cells that depend on persisting antigen for their continuous renewal, either by reactivation of memory $T$ cells and/or priming of recent thymic emigrants. At the time of pathogen penetration into localized tissue (for example, liver or skin), only vaccines that have maintained a population of rapidly recruited or tissue-resident effector T cells confer adequate protection.

efficacy of the subunit vaccines approached the potency and durability of the protection conferred by live or live-attenuated whole-organism vaccines. The feasibility of developing a live, attenuated vaccine using irradiated, asceptic, cryopreserved sporozoites manually dissected from mosquito salivary glands and delivered intravenously by needle was revisited starting around 10 years ago ${ }^{6}$. The most recent studies using this vaccine revealed that high doses (>600,000 sporozoites) completely protected six of six volunteers against infectious sporozoite challenge ${ }^{8}$. Although these numbers are small, and the high dose and route of inoculation pose substantial challenges moving forward, this trial still represents the most efficacious outcome of a malaria vaccine in humans.

Individuals that received the highest dose of sporozoites and who were most protected had higher liver stage-specific antibodies, and both $\mathrm{CD}^{+}$and $\mathrm{CD}^{+} \mathrm{T}$ cell responses. The tissueinfiltrating or resident memory cells in the liver most relevant to the immune status of these individuals could not be studied. In nonhuman primates and mice immunized with irradiated sporozoites, high numbers of liver stage-specific $\mathrm{CD}^{+} \mathrm{T}$ cells were found predominantly in the liver and were required for sterile immunity 9 . Mouse studies have revealed a requirement for $\mathrm{CD} 4^{+} \mathrm{T}$ cells and antigen persistence to generate and maintain a protective $\mathrm{CD} 8^{+} \mathrm{T}$ cell response $\mathrm{e}^{9}$. Treatment with primaquine, which eliminates the nondividing exoerythrocytic forms that have been observed in mice immunized with irradiated or genetically attenuated sporozoites, completely abolished the long-lived protection conferred by these vaccines ${ }^{10}$. In other studies, liver-stage remnants were not observed, and the protection conferred by irradiated sporozoites was not sensitive to treatment with primaquine, but the conclusion was still that a source of persisting antigen is required based on the finding that circumsporozoite protein-specific $\mathrm{CD} 8^{+}$ $\mathrm{T}$ cells could be primed when transferred in mice that had been immunized up to 60 days earlier ${ }^{11}$. In mice immunized with genetically attenuated sporozoites ${ }^{12}$ short-lived effector $\mathrm{CD}^{+} \mathrm{T}$ cells $\left(\mathrm{KLRG}^{+} \mathrm{CD} 127^{-}\right)$present before challenge were responsible for the rapid clearance of infected hepatocytes and presumably required some form of persisting antigen for their renewal.

\section{The durability of the protective response}

If antigen persistence is necessary to maintain the sterilizing immunity observed in attenuated-sporozoite vaccines and if immunological memory, as conventionally defined, is maintained by long-lived memory cells in the absence of antigen, then what, if any, is the role of memory $\mathrm{T}$ cells in these vaccines? As some cells in the effector-memory cell pool, especially $\mathrm{CD}^{+}{ }^{+} \mathrm{T}$ cells ${ }^{13}$, need continual reminding by antigen, their distinction from short-lived, fully differentiated effector $\mathrm{T}$ cells seems a semantic point. Operationally, the critical parameter is how quickly the protective response decays in the absence of antigen. It is not yet known whether the long-lived protection elicited by irradiated sporozoites in people is dependent on persisting antigen. An alternative approach based on using live, whole organisms involved exposure to $P$. falciparum-infected mosquitoes followed by chloroquine treatment to kill blood-stage parasites provided long-lasting sterile immunity in volunteers ${ }^{14}$, but it is not known how long liver-stage antigens persisted in these infected individuals to maintain the protective response.

In mice, the requirement for antigen persistence can be more carefully addressed by adoptive transfer of the protective cells into naive recipients and by delaying the time between the transfer and challenge. Using this approach, it was established unequivocally that long-lived, memory $\mathrm{CD}^{+} \mathrm{T}$ cells could mediate protection against lymphocytic choriomeningitis virus (LCMV), as both the numbers and protective function of the virus-specific cells were maintained when challenge was delayed for up to 18 months after transfer ${ }^{15}$. The interval between adoptive transfer and challenge has so far been extended to only a few days using the protective cells generated by sporozoite vaccines, so the lifespan of the protective cells in the absence of antigen is still not clear even in the mouse models. The closest that any malaria vaccine has come to reproducing the LCMV results in mice is the long-lived, sterile immunity achieved using a prime-boost strategy involving circumsporozoite peptide-coated dendritic cells followed by treatment with Listeria monocytogenes expressing the same peptide ${ }^{16}$. Although the lifespan of the protective $\mathrm{CD} 8^{+} \mathrm{T}$ cells was not defined by adoptive-transfer studies, the nature of the vaccine would suggest that immunity did not depend on persisting antigen. Similar to the case with LCMV, and distinct from vaccination with attenuated sporozoites, the peptide-specific, prime-boost vaccine may bypass the requirement for help of $\mathrm{CD} 4^{+} \mathrm{T}$ cells and persistence of antigen to maintain the protective response. The generation of a protective population containing bone fide effectormemory $\mathrm{CD}^{+} \mathrm{T}$ cells may depend on the increased clonal expansion and signal strength associated with the activation of the peptidespecific cells. It is not clear how relevant or practical this prime-boost, epitope-based approach is to human vaccination. A heterologous primeboost vaccine designed to generate high numbers of $\mathrm{CD}^{+} \mathrm{T}$ cells specific for the liver-stage antigen ME-TRAP induced some protection in over half of the 14 volunteers but sterile immunity in only three of them ${ }^{17}$.

Live vaccination against Leishmania major For cell-mediated immunity against pathogens that reside in phagosomes, for example, 
Mycobacterium, Salmonella and Leishmania spp., the need to recognize peptides that are generated in endosomal compartments dictates that $\mathrm{CD} 4^{+} \mathrm{T}$ cells will be a critical component of the protective response. There is clear evidence that antigen persistence is necessary to maintain this response $\mathrm{e}^{18}$, and is the essential quality of the $L$. major vaccine that provides strong and long-lasting protection against cutaneous leishmaniasis in people. Deliberate needle inoculation with viable $L$. major parasites in a selected site, referred to as 'leishmanization', has been used for generations as a live 'vaccine' in people living in regions endemic for cutaneous forms of leishmaniasis and provides long-lasting protection against natural exposure ${ }^{19}$. Because the live organisms used have not been deliberately attenuated, this is not a vaccine in the conventional sense. Nonetheless, the inoculum is effectively attenuated because it contains cultured organisms that are less virulent than the forms inoculated by the sand-fly vector and because the parasites are delivered without the factors co-egested by the sand fly, which are known to promote infection. That said, the practice has been largely discontinued, partly because of the severity of the primary lesions experienced by some vaccinees. Many attempts to reproduce this protection using whole-cell killed vaccines, including formulations shown to work effectively as immunotherapy, have failed to confer significant protection against natural exposure in multiple phase III trials ${ }^{20}$. Thus, leishmanization remains the only effective prophylactic vaccine in people, and irradiated or genetically attenuated organisms could be used to address the safety concerns.

In mice, as in people, a primary infection with L. major that heals represents the gold standard of acquired resistance, unequaled by a variety of experimental whole-cell killed and subunit vaccines that have been evaluated, some of which have failed entirely when tested under the more stringent conditions of challenge with infected sand flies ${ }^{21}$. The acquired resistance observed in healed mice is sometimes referred to as 'concomitant immunity', meaning that the protection against reinfection coincides with the persistence of the primary infection. Despite this persistence, the cells mediating resistance to reinfection do not become exhausted; on the contrary, long-lived protection from secondary challenge is lost if the original infection is cleared $^{22}$.

The rapid recruitment of IFN $-\gamma^{+} \mathrm{CD} 4^{+} \mathrm{T}$ cells to the cutaneous site of a bite by infected sand fly or needle injection is the strongest correlate of protection against reinfection with $L$. major in healed mice ${ }^{21}$. Adoptive transfer of polyclonal, antigen-experienced $\mathrm{T}$ cell populations from healed mice into naive recipients has confirmed that $\mathrm{CD} 4^{+} \mathrm{T}$ helper type $1\left(\mathrm{~T}_{\mathrm{H}} 1\right)$ cells present in both the central and effector memory pools are sufficient to transfer protection against needle challenge ${ }^{23}$. In more recent studies, only cells bearing markers of terminally differentiated $\mathrm{T}_{\mathrm{H}} 1$ effector cells $\left(\mathrm{CD} 44^{+} \mathrm{CD} 6 \mathrm{~L}^{-} \mathrm{T}^{-}\right.$bet $^{+} \mathrm{Ly}_{6 \mathrm{C}^{+}}$) were rapidly recruited to the site of cutaneous challenge, and these cells were required to fully reconstitute the protective response (N. Peters, A. Pagan, P. Lawyer, T. Hand, E. Roma, L. Stamper et al.; unpublished data). Most importantly, if L. major challenge was delayed by only 14 days after transfer, no recruitment of $\mathrm{T}_{\mathrm{H}} 1$ effector cells was observed, and immunity was lost. Thus, concomitant immunity is mediated by a population of preexisting and shortlived effector T cells, which is maintained as a stable population by the persisting infection, and continuously renewed by the activation of recent thymic emigrants and/or by the periodic restimulation and differentiation of memory cells.

\section{Zinkernagel's edict}

Concomitant immunity is thus a dynamic state, and memory cells undoubtedly contribute to its maintenance. But their generation is not a sufficient condition for protection; succinctly put by Zinkernagel ${ }^{24}$, memory does not equal protection. Just as the best correlate of protection against extracellular infections is the level of preexisting neutralizing antibody, in Leishmania infection or intracellular stages of malaria, the best correlate is the number of effector $\mathrm{T}$ cells present at the time of exposure (Fig. 1). Like antibodies, effector T cells perform critical peripheral immune-surveillance functions. They would be expected to have a decided advantage in localized pathogen control over central memory $\mathrm{T}$ cells, whose immune functions are delayed by the requirement to undergo antigen-stimulated expansion, differentiation and trafficking. In this context, tissue-resident memory $\mathrm{T}$ cells would be expected to possess the most immediate capacity for localized control of infection. Such resident memory cells function in persistent viral infections to limit the establishment of new infections, particularly in the skin ${ }^{25}$. It is still not clear, however, whether persisting infection is required to maintain their long-term tissue residence, whether memory $\mathrm{CD} 4^{+} \mathrm{T}$ cells establish tissue residence, or more generally, whether resident memory $\mathrm{T}$ cells contribute to concomitant immunity in chronic parasitic infections.

The difficulties associated with live, wholeorganism vaccines are clear and present, and continued priority should be given to clinical trials of defined, subunit vaccines against human parasitic diseases, especially those already in the development pipeline. But the exceptional outcomes of live, whole-organism vaccines against human malaria and leishmaniasis, and the experimental models that have revealed the requirement for persisting, whole organismderived antigens to drive the breadth and rapidity of the localized protective response suggest that there may be no adequate replacement for approaches based on live, and live-attenuated, whole organisms. Rather than endure the long wait for the outcome of clinical trials of subunit vaccines to be known, and so long as safety concerns are fully met, these approaches should be pursued as a parallel effort at least.

\section{ACKNOWLEDGMENTS}

I thank B. Seder and N. Peters for helpful discussions. This work was supported by the Intramural Research Program of the US National Institute of Allergy and Infectious Diseases, National Institutes of Health.

\section{COMPETING FINANCIAL INTERESTS}

The author declares no competing financial interests.

Reprints and permissions information is available online at http://www. nature.com/reprints/index.html.

1. Beaumier, C.M., Gillespie, P.M., Hotez, P.J. \& Bottazzi, M.E. Transl. Res. 162, 144-155 (2013).

2. Hotez, P.J. \& Ferris, M.T. Vaccine 24, 5787-5799 (2006).

3. Sacks, D.L., Sher, A., Riley, E.M. \& Wynn, T.A. in Fundamental Immunology 6th edn. (ed., Paul, W.E.) 1097-1130 (Lippincott, Williams \& Wilkins, 2008).

4. Clyde, D.F., Most, H., McCarthy, V.C. \& Vanderberg, J.P. Am. J. Med. Sci. 266, 169-177 (1973).

5. Nussenzweig, R.S., Vanderberg, J., Most, H. \& Orton, C. Nature 216, 160-162 (1967).

6. Luke, T.C. \& Hoffman, S.L. J. Exp. Biol. 206, 3803 3808 (2003).

7. Hill, A.V. Philos. Trans. R. Soc. Lond. B Biol. Sci. 366 2806-2814 (2011).

8. Seder, R.A. et al. Science 341, 1359-1365 (2013).

9. Tse, S.W., Radtke, A.J. \& Zavala, F. Mem. Inst. Oswaldo Cruz 106 (suppl. 1), 172-178 (2011)

10. Scheller, L.F. \& Azad, A.F. Proc. Natl. Acad. Sci. USA 92, 4066-4068 (1995).

11. Cockburn, I.A. et al. PLoS Pathog. 6, e1000877 (2010).

12. Cooney, L.A. et al. Infect. Immun. 81, 4171-4181 (2013).

13. Homann, D., Teyton, L. \& Oldstone, M.B. Nat. Med. 7 913-919 (2001).

14. Roestenberg, M. et al. Lancet 377, 1770-1776 (2011).

15. Lau, L.L., Jamieson, B.D., Somasundaram, T. \& Ahmed, R. Nature 369, 648-652 (1994).

16. Schmidt, N.W. et al. Proc. Natl. Acad. Sci. USA 105 14017-14022 (2008)

17. Ewer, K.J. et al. Nat. Commun. 4, 2836 (2013).

18. Nelson, R.W., McLachlan, J.B., Kurtz, J.R. \& Jenkins, M.K. J. Immunol. 190, 2828-2834 (2013).

19. Khamesipour, A. et al. Vaccine 23, 3642-3648 (2005).

20. Noazin, S. et al. Vaccine 26, 6759-6767 (2008).

21. Peters, N.C. et al. PLoS Pathog. 5, e1000484 (2009).

22. Belkaid, Y., Piccirillo, C.A., Mendez, S., Shevach, E.M. \& Sacks, D.L. Nature 420, 502-507 (2002).

23. Zaph, C., Uzonna, J., Beverley, S.M. \& Scott, P. Nat. Med. 10, 1104-1110 (2004).

24. Zinkernagel, R.M. Cell. Mol. Life Sci. 69, 1635-1640 (2012).

25. Gebhardt, T., Mueller, S.N., Heath, W.R. \& Carbone, F.R. Trends Immunol. 34, 27-32 (2013). 